\title{
Numerical analysis of the hydrodynamic ram of a CFRP integral tank
}

\author{
P. Stephani ${ }^{1}$, P. Middendorf ${ }^{2} \&$ Ch. Le $\beta^{3}$ \\ ${ }^{1}$ University of Stuttgart, IFB, Germany \\ ${ }^{2}$ EADS Corporate Research Center Germany, LG-CT \\ ${ }^{3}$ EADS Military Air Systems, OPES1, Germany
}

\begin{abstract}
The vulnerability of a CFRP integral tank against HRAM (hydrodynamic ram) is analysed. The geometry of the tank is based on a generic MALE (Medium Altitude Long Endurance) UAV (Unmanned Aerial Vehicle). Several designimprovements are to be analysed. HRAM occurs if a high-energetic object penetrates a fluid filled structure (e.g. a fuel tank below its fill level). Pressure waves, originating from the impactor, cause severe pressure loads on the walls. This can lead to the destruction of the tank and in case of an integral tank, to the failure of structural necessary components. One segment of the integral tank from the generic MALE-wing is simulated using the explicit finite element code LS-DYNA with Fluid-Structure-Interaction. The analysed design-solutions include spars with holes in it that should work as pressure outlets and a redesign of the outer spar.
\end{abstract}

Keywords: HRAM, hydrodynamic ram, UAV, MALE, integral tank, LS-DYNA, CFRP, FSI, fluid structure interaction.

\section{Introduction}

HRAM poses a significant threat to modern military aircraft, since the fuel tanks represent the largest exposed area of all vulnerable components. Data from "Desert Storm" shows that $75 \%$ of all aircraft losses can be traced back to the fuel system (Addessio [1]). These losses can be attributed to three main effects: fire, explosion and HRAM. Statistics indicate that HRAM is by far the most lethal of these three. This is especially true for highflying UAVs, having a large wing area because of aerodynamic requirements. These aircraft-types feature 
high aspect ratio wings and therefore a small chord. This in turn leads to a wing design that cannot use a multispar-concept and therefore has little resistance against HRAM. The effects of HRAM are especially devastating in case of CFRP structures, since this type of material cannot undergo any significant plastic deformation. Therefore its ability to absorb energy without failure is very limited. Furthermore, HRAM poses a severe risk especially for aircraft employing extreme lightweight design-philosophies. HRAM is particularly lethal for aircraft-types, which incorporate all of the above illustrated features, like MALEs. The vulnerability of aircraft against HRAM is, however, not limited to military types. In 2000, a Concorde crashed shortly after takeoff from Charles de Gaulle (France). The following investigations showed that HRAM had played a significant role in the loss of the aircraft.

Design concepts that lead to a better resistance of the structure against HRAM could either be based on strengthening the airframe to such an extent that it can withstand HRAM loads, or to use structural elements that intentionally fail under well defined circumstances and in doing so, absorb or redirect HRAMenergy. The first concept, however, contradicts the requirements of extreme lightweight design-philosophies. Therefore the idea of an improved design is based on the creation of intentionally weak areas. They have to withstand loads experienced during normal flight operations, but must fail quickly enough under HRAM to release or absorb pressure before it leads to severe damage in structurally significant parts. This design basically resembles overpressure valves.

EADS military performed a threat analysis for a typical MALE missionprofile. Among definitely catastrophic threats like SAM, a .50 AP bullet, impacting at $560 \mathrm{~m} / \mathrm{s}$, was identified as a realistic threat, resulting in a probably survivable impact energy of $7 \mathrm{~kJ}$ (Leß [5]). The projectile impacts in the tank segment near the leading edge, nearly perpendicular to the flight-direction and from below.

\section{HRAM}

Hydrodynamic ram consists of three sequential phases. The shock phase, the drag phase and the cavitation phase. Each of the three phases contributes to structural damage in a different way and extent.

The shock phase is initiated when the impactor penetrates the wall of the fluid filled structure. Impact energy is transferred to the fluid and hemispherical shock wave is formed. This leads to damage primarily in the vicinity of the impact position. Further away, the effect on the structure is very small, since the pressure in the shockwave is proportional to the shock area and therefore decreases by $1 / \mathrm{r}^{2}$. The pressure in the shockwave can reach values of up to 500bar, depending on the boundary conditions and lasts for several microseconds.

During the drag phase, the kinetic energy of the impactor is partially transformed into fluid motion. The displacement of the fluid along the impactors path leads to a radial pressure field. In contrast to the pressure field generated 
during the shock phase, the fluid is accelerated rather slowly. This leads to much lower pressure values, however, they last much longer. The typical values lie in the range of 10 to 50bar, with a duration of approx. $1 \mathrm{~ms}$, again depending on the boundary conditions. The damage caused by the drag phase is not restricted to the impact point but can affect the whole fluid filled structure.

The displacement of fluid during the shock phase forms a cavity along the impactors path. The following expansion und collapse (oscillating) of the cavity is named cavity-phase and can cause further significant pressure pulses. Figure 1 shows a typical HRAM pressure load experienced near the point of impact.

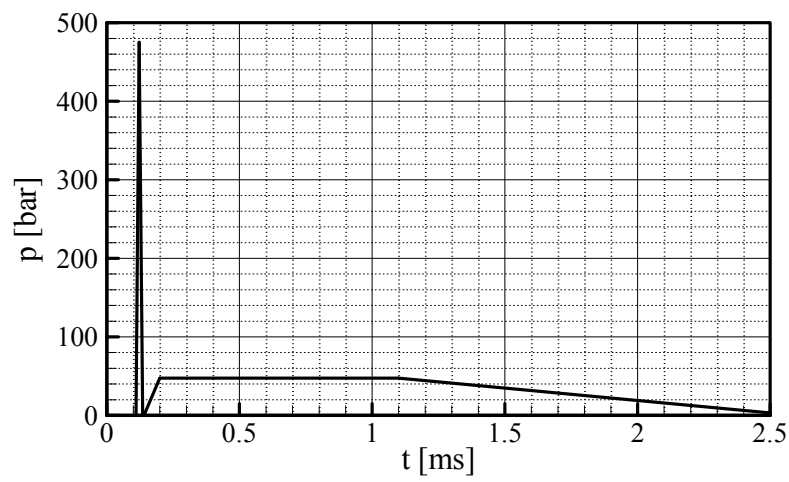

Figure 1: Typical HRAM pressure loads.

\section{Design concepts}

The basic layout of the wing-segment is depicted in Figure 2. The wing-design features a sandwich skin that consists of CFRP skins and a foamcore (yellow), rips and 3 spars, all made of CFRP. For further information refer to Bauer [4].

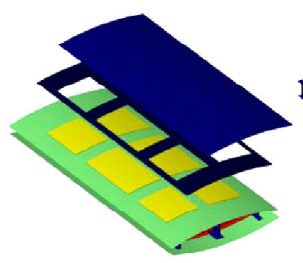

a

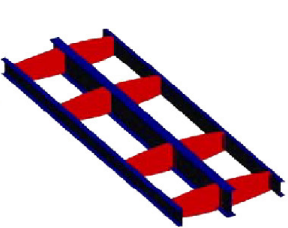

b

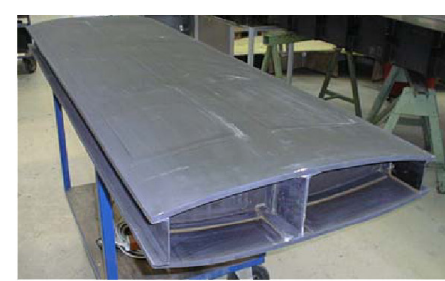

c

Figure 2: $\quad$ MALE wing-segment: Sandwich-skin (a), Spar and Rips (b), actual hardware(c). 
These parts are produced using VAP (Vacuum Assisted Process), a special infiltration process developed by EADS (see Bauer and Körwien [3]). Afterwards, the rips, spars and the skins are joined together using adhesive bonding.

Following design-considerations that did not include HRAM-loads, instead are exclusively based on static and dynamic loads experienced during normal flight operations, the C-spars openings are facing outwards (see Figure 3). For the reminder of this paper, the described design will be referred to as "reference design".

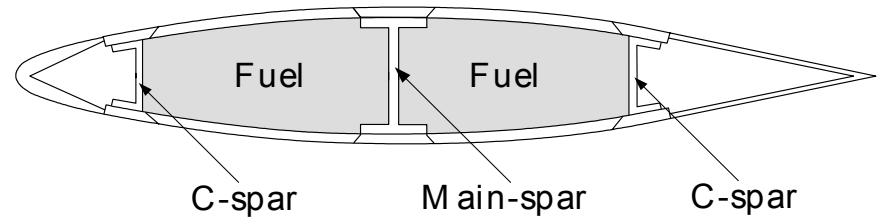

Figure 3: $\quad$ Cross-section of the wing.

\subsection{Improved design}

The already mentioned overpressure-valves are implemented by cutting holes in the C-spars. Afterwards these holes are closed with a membrane consisting of sheetmetal or elastomer-plates. Two concepts are available: Putting the membrane on the inside of the tank (see Figure 4, left) leads to the so-called membrane-configuration. If the membrane is bonded to the outside of the tank, this design is referred to as plate-configuration. In the plate-configuration the bonding has to fail, whereas in den membrane configuration the membrane itself must fail.

If the pressure in the fuel arises (e.g. during a HRAM event), the current orientation of the $\mathrm{C}$-spars will lead to a high peel-load in the bondings between the $\mathrm{C}$-spars and the skin. Therefore the orientation of the $\mathrm{C}$-spars is reversed, so that they open inwards. This should decrease the peel-load.
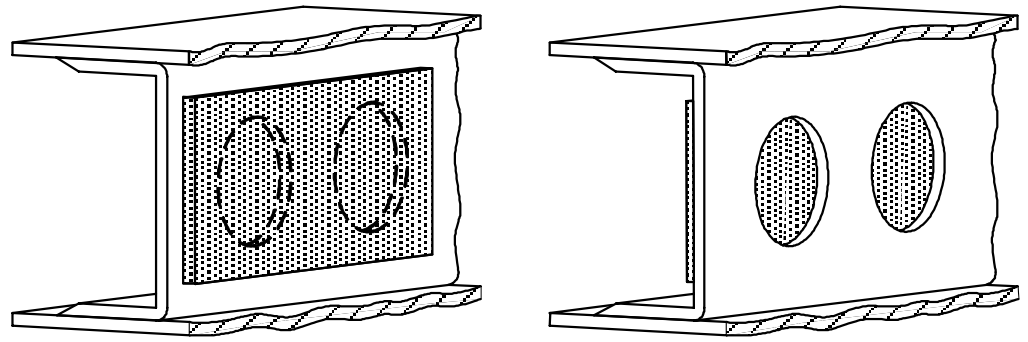

Figure 4: $\quad$ Membrane- and plate-configuration (right). 


\section{Model}

Figure 5 shows the simulated segment of the MALE-integral-tank. As specified by EADS military, it is the segment closer to the leading edge. The model of the integral-tank-segment includes the exact CFRP-layer-configuration and sandwich structures with foam-core. Both, CFRP and foam are modeled using the Material Model 54. The sandwich layout is specified with the help of userdefined integration. The Failure-criteria used is based on EFS (Effective Failure Strain). Several bonded-joints are simulated by using a special purpose, noncommercial, delamination contact model. For further information regarding this contact see Borg [2]. The tank-components consist of approx. 25,000 Belytschko-Lin-Tsay-type shell elements (LS-DYNA type 2), see Figure 6, left. For clarification, the top skin is not shown in the figure. The elements measure 3 to $8 \mathrm{~mm}$.

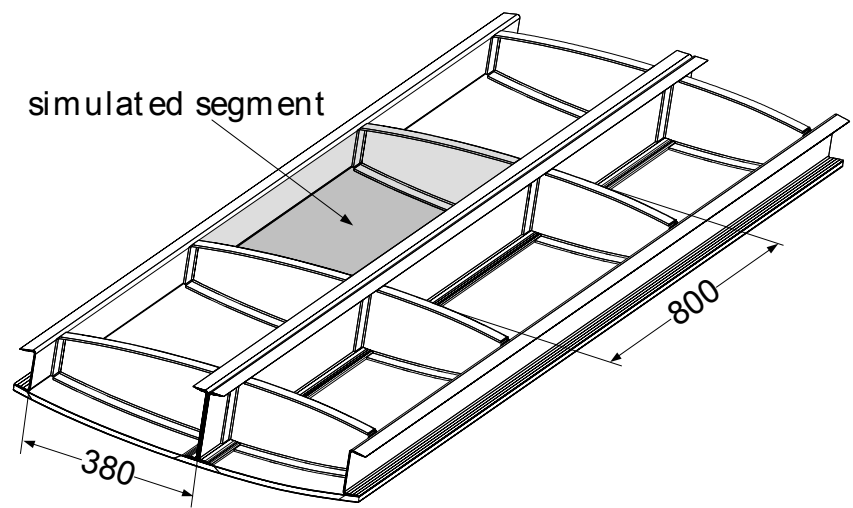

Figure 5: $\quad$ Simulated segment, dimensions in $\mathrm{mm}$.

The projectile is modeled as rigid body, insofar the expected deformation is small and for this simulation not of particular interest. Rigid bodies have a great advantage over ones modeled using very stiff materials insofar that the size of the elements they are consisting of, do not affect the time step. This is especially important in this case, since a good discretisation of the projectile requires quite small elements (see Figure 6, right). The impactor consists of 60 Hughes-Liuvolume-elements (LS-DYNA type 1)

The fuel is modeled as water, using a polynomial equation of state. This is justified by the fact that the pressures involved in this simulation are in the range of several 1000bar and therefore do not require a Grüneisen-equation or the like. The fluid consists of approx. 500,000 ALE multimaterial elements (LS-DYNA type 11). The element size varies from $3 \mathrm{~mm}$ in the region along the anticipated projectile path, to $10 \mathrm{~mm}$ on the side at the outer extents of the fluid domain.

Between the impactor and the skins an antipenetration contact is defined. A Eulerian-Lagrangian penalty-based coupling algorithm is used to simulate the interaction between the projectile/tank (Lagrangian) and the fluid (Eulerian). The 
reason why the penalty-based method is chosen in favor of constraint-based techniques is that it results in a significantly smaller loss of kinetic energy at coupling interfaces. The numerical loss of kinetic energy should be kept at a minimum considering its importance in the present case.
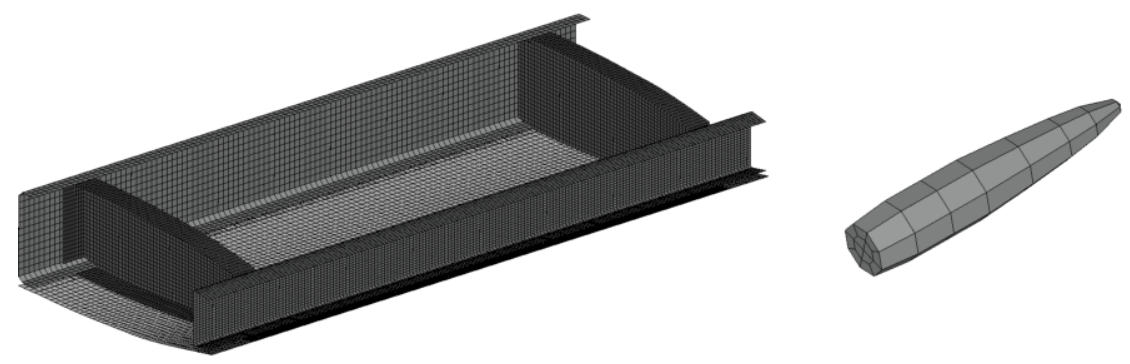

Figure 6: $\quad$ FE-model of the tank and projectile.

\section{Results}

The level of damage cause to the structure through the HRAM pressure heavily depends on the tumbling of the projectile. Depending on the boundary conditions, the behavior of the bullet and therefore the damage is quite different. Figure 7 shows a typical tumbling of the projectile and the resulting cavity.

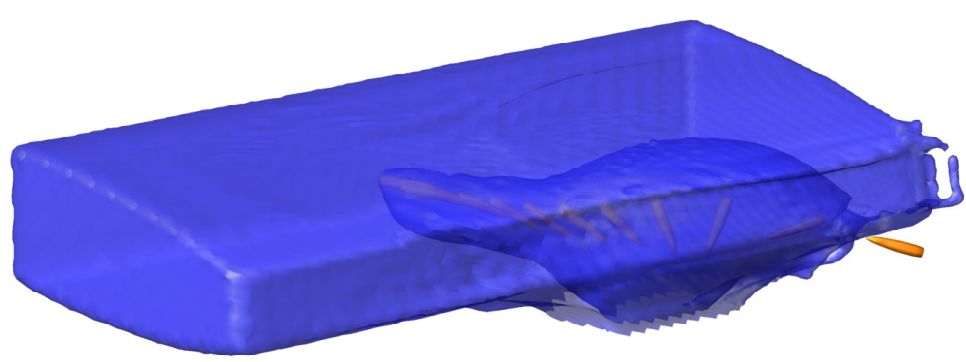

Figure 7: $\quad$ Projectile path and cavity $(\Delta \mathrm{t}=0.2 \mathrm{~ms})$.

The following pictures illustrate the damage caused to the structure and to the bondings for different configurations. It should be noted that the damage is not necessarily fully developed at the depicted times. The level of damage, however, would not significantly rise further and the simulations were therefore cut short.

In the reference design, the skin fails on a large area and the C-spar is torn open in wingspan direction (see Figure 8). The bondings fail between the skin and the C-spar and even between the C-spar and the rips. In Figure 9, the 
improvement caused by the reversed $\mathrm{C}$-spar is clearly visible. The overall damage to the C-spar is smaller compared to the reference design. Nevertheless, the damage to the skin is more or less of the same level. As to be expected, the peel load is definitely smaller and therefore there exists no significant failure in the bonding between the skin and the C-spar. Additionally, the bonding between the rips and the $\mathrm{C}$-spar nearly remains intact. Altogether, the reversed C-spar shows a clear improvement to the reference design.

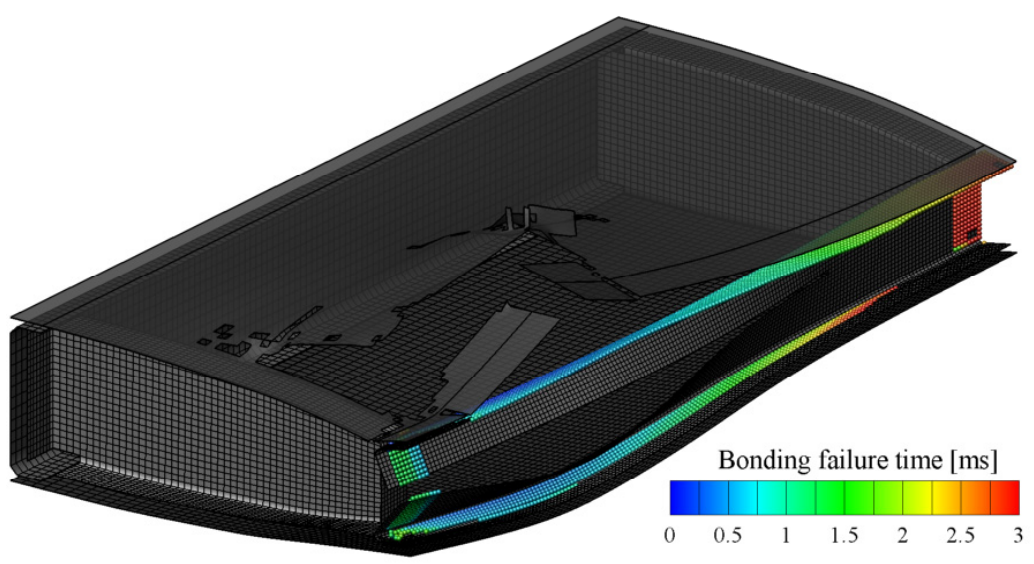

Figure 8: $\quad$ Damage after $\mathrm{t}=3 \mathrm{~ms}$ (reference design).

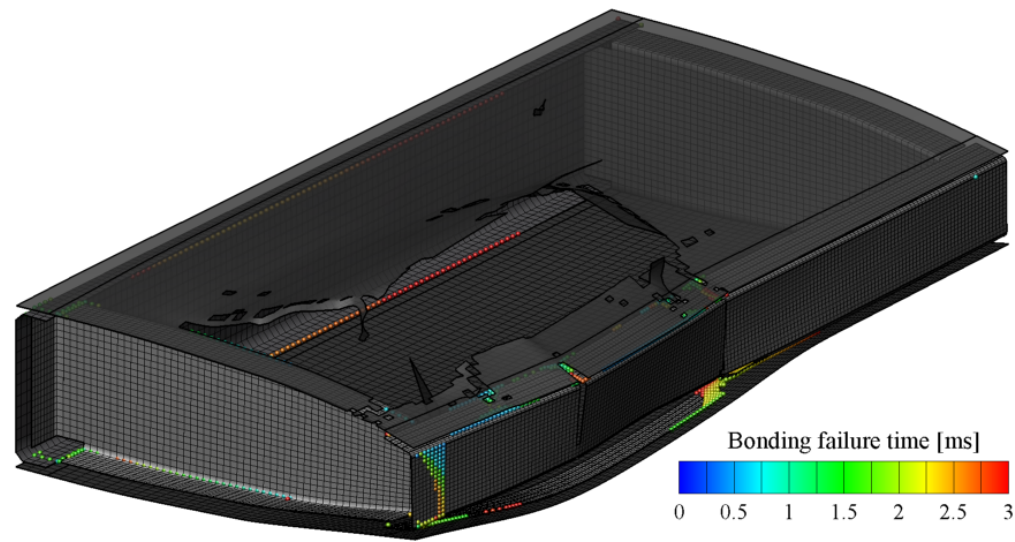

Figure 9: $\quad$ Damage after $\mathrm{t}=3 \mathrm{~ms}$ (reversed C-spar).

The following figures show the design with holes in the C-spar compared to one with a homogenous spar. Both designs feature a reversed spar, so that a possible difference between these two concepts can distinctively be attributed to the 
holes. However, there is not much of a difference between these two concepts (see Figures 10 and 11). Only the C-spar is marginally less deformed in case of the design with the hole. Obviously, the holes lead to a local reduction of the loads against the structure, particularly the $\mathrm{C}$-spar. The explanation of this effect is quite simple. Because of the holes in the C-spar, the area on which the pressure can act is smaller and therefore the loads, too.

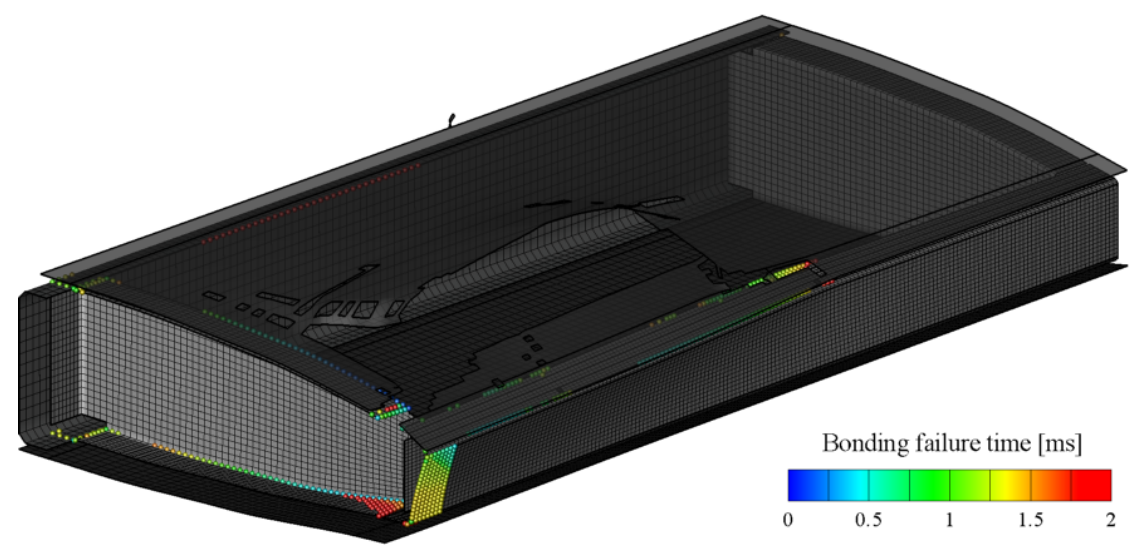

Figure 10: Damage after 2ms (homogenous spar).

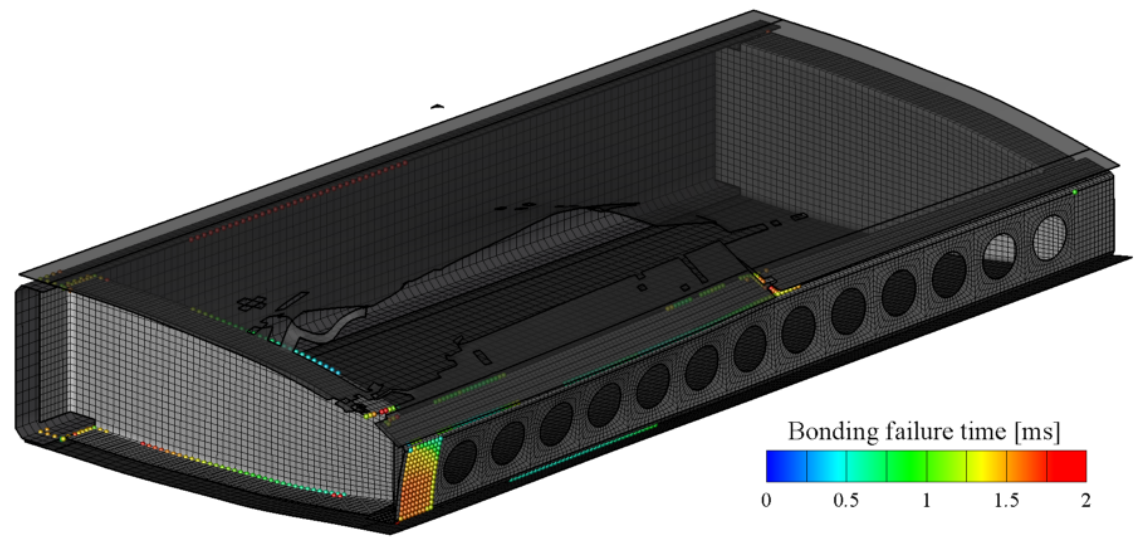

Figure 11: Damage after 2ms (spar with holes).

However, the intended effect of a global reduction of the HRAM loads cannot be observed; only the pressure levels far from the impact point are marginally lower (see Figure 12). The pressure load near the point of impact fit very well to the one derived from experiments (comp. Figure 1 and Figure 12, left). 


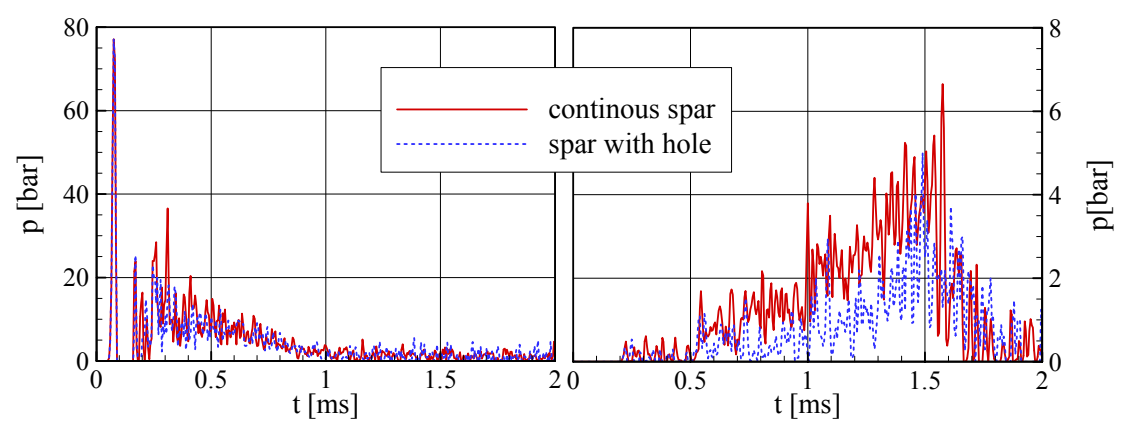

Figure 12: $\quad$ Pressure near (left) and far from the point of impact (right).

\section{Conclusions}

A qualitative simulation von HRAM-effects in a CRFP-structure with LS-DYNA seems to be possible. Whether the simulation shows a quantitative agreement, remains to be seen.

The displacement of fluid during the so-called "Drag Phase" can be identified as the primary cause of damage. The damage-level of a tumbling projectile is significantly higher compared to that of a stabilized impactor.

The simulations show that the pressure-outlets cannot significantly increase the resistance of the structure against HRAM. The improvements are restricted to a smaller impulse-load against the spar and have no global effect. The reversed spar, however, improves the survivability of the structure. But even for the improved design, the energy level of current ballistic parameters still might cause total destruction of the tank.

\section{References}

[1] Addessio, F.L. et al., Physics-Based Damage Predictions For Simulating Testing and Evaluation Experiments, LA-UR-97-4877, Los Alamos National Laboratory, 1997.

[2] Borg, R., Implementation of a Tie-Break Interface into LS-DYNA, Linköping, 2003.

[3] Bauer, J., Körwien T., Next Generation of Composite Manufacturing Technology, DGLR Jahrestagung, Hamburg, Sept. 2001.

[4] Bauer, J., Strukturelemente für Unmanned Air Vehicles aus faserverstärkten Kunststoffen, EADS-S-R-1942; E /0D038/M6202, 2004.

[5] Leß, Ch., MALE UAV Vulnerability, EADS-S-R-1997, EADS Military Air Systems, Germany, 2005. 